\title{
Schriftraum Diaspora: Die moderne Ästhetik der ewigen Wanderschaft bei Stefan Zweig
}

Paola Paumgardhen, L'espace scriptural de la diaspora : l'esthétique moderne de l'errance éternelle chez Stefan Zweig

\section{Paola Paumgardhen}

\section{OpenEdition}

\section{Journals}

Édition électronique

URL : https://journals.openedition.org/austriaca/873

DOI : 10.4000/austriaca.873

ISSN : 2729-0603

\section{Éditeur}

Presses universitaires de Rouen et du Havre

Édition imprimée

Date de publication : 1 décembre 2019

Pagination : 211-233

ISBN : 979-10-240-1454-8

ISSN : 0396-4590

\section{Référence électronique}

Paola Paumgardhen, „Schriftraum Diaspora: Die moderne Ästhetik der ewigen Wanderschaft bei Stefan Zweig", Austriaca [Online], 88-89 | 2019, Online erschienen am: 31 Dezember 2020, abgerufen am 24 Oktober 2022. URL: http://journals.openedition.org/austriaca/873 ; DOI: https://doi.org/ 10.4000 /austriaca. 873 


\section{Schriftraum Diaspora: Die moderne Ästhetik der ewigen Wanderschaft bei Stefan Zweig}

In den Zwanzigerjahren besuchte Stefan Zweig eines Tages mit dem Berliner Dramatiker Otto Zarek eine Ausstellung antiker Möbel in München. Beim Anschauen etliche Holztruhen konnte der feinsinnige Beobachter unterscheiden, welche Juden gehört hatten. „Sie haben unten Rollen“ - erklärte Zweig lächelnd - „Damals - wie eigentlich immer konnten die Juden nie sicher sein, wann die Pfeife ertönte, wann das Rasseln der Pogrome näher kam. Sie mussten bereit sein, von einem Augenblick zu dem anderen das Weite zu suchen. [...] Ja, diese Truhen auf Rollen sind bemerkenswerte Sinnbilder für das jüdische Schicksal!"

Der Schriftsteller, der in einer assimilierten jüdischen Familie im Zentrum der Österreichisch-Ungarischen Monarchie geboren wurde und in der kosmopolitischen Stadt Wien aufgewachsen war, nahm gegenüber der erzählten Anekdote des jüdischen Exils nur scheinbar eine distanzierte und überblickende Außenperspektive ein. Zweigs europäisches Selbstverständnis ist nämlich von dem Bewusstsein seiner jüdischen Identität nicht zu trennen. Obwohl er sein Leben lang dem alten multikulturellen und toleranten Wien - „nirgends war es leichter, ein Europäer zu sein“2 - sein Weltbürgertum und seinen humanistischen Geist zuschrieb, war seine Verwurzelung im Judentum weder oberflächlich noch sekundär. Bereits bei dem jungen Zweig muss es ein mehr oder weniger bewusstes Gefühl gegeben haben, Teil der Diaspora zu sein. Dass der Autor mit dem Judentum eng verbunden war und dass er sich mit der Geschichte und dem Geist seiner Glaubensbrüder identifizierte, drücken seine eigenen Worte überzeugend aus: „[...] noch geistern die zweitausend Jahre Wanderschaft vorzeitiger

1. Zit. nach George Prochnik, Das unmögliche Exil. Stefan Zweig am Ende der Welt, Übers. Andreas Wirthensohn, München, C. H. Beck, 2016, S. 148-149.

2. Stefan Zweig, Die Welt von Gestern. Erinnerungen eines Europäers, Frankfurt/M., Fischer Verlag, 2016, S. 40. 


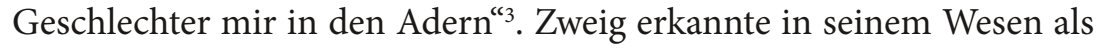
einziges jüdisches Erbteil „den Mangel an Heimatgefühl“4, das ihn keine „Grenzen innerhalb Europas“5 empfinden ließ, so dass er sich „in Paris wie in Rom wie in Deutschland"“6 gleichermaßen zu Hause fühlte. Dies lag, wenn man seinen Erinnerungen in der Welt von Gestern (1942) glaubt, an der humaneren Stimmung der Donaustadt, die trotz eines langsam erstarkenden Antisemitismus unter Karl Lueger noch zu spüren war ${ }^{7}$. Bis auf den heutigen Tag ist die Frage umstritten, ob Zweig ein „Homo Judaicus“ war, da er - so Harry Zohn - lange eher als „ein guter Europäer“ [denn] „als ein guter Jude“" gelebt hat. Da Zweigs Bild - wie Mark H. Gelber richtig feststellt - in verschiedenen Biographien zum größten Teil von seinen spezifisch jüdischen Aspekten gereinigt war ${ }^{9}$, ist es wenig verwunderlich, dass die europäische Identität des Schriftstellers meistens stärker betont ist als seine jüdische Zugehörigkeit. Einige Zweig-Forscher vertreten dagegen die Ansicht, dass Zweigs Europäertum als Reaktion auf bestimmte historische, politische und auch kulturelle Ereignisse seiner Epoche anzusehen ist. Margarita Pazi betrachtet Zweigs humanistischen Kosmopolitismus als „Antwort auf das Gefühl des 'Nicht-ganz-Dazugehörens"“10, also eben jener Diaspora, die der akkulturierte Wiener Jude noch nicht bewusst reflektierte, aber doch

3. Stefan Zweig, „Das Jüdische in meinem Wesen und Schaffen“, in Bayerische Israelitische Gemeindezeitung 8 (1926), S. 228-230, hier S. 230.

4. Ebd.

5. Ebd.

6. Ebd.

7. Jacques Le Rider betont, dass die jüdischen Intellektuellen der älteren Generation die antisemitischen Unruhestifter (wie Lueger) oft kaum ernst nahmen. Diese Unterschätzung der wirklichen Gefahr des Wiener Antisemitismus war ein Kennzeichen der liberalen alten Garde. Vgl. Jacques Le Rider, „Stefan Zweig - Darstellung des Judentums in den Erzählungen der dreißiger und vierziger Jahre“, in Mark H. Gelber, Klaus Zelewitz (Hg.), Stefan Zweig. Exil und Suche nach dem Weltfrieden, Akten des Internationalen Stefan Zweig-Kongresses, 18.-23.2.1992, Schloss Leopoldskron Salzburg, Riverside, Adriane Press, 1995, S. 206.

8. Harry Zohn, „Jewish Themes in Stefan Zweig“, in Journal of the International Arthur Schnitzler Research Association, 6, 2 (1967), S. 36.

9. Gelber ist der Meinung, dass die erste Generation der Zweig-Forscher, vor allem die erste Frau von Stefan Zweig, Friderike von Winternitz (Stefan Zweig, Wie ich ihn erlebte, 1948), und der Zweig-Biograph Donald Prater (European of Yesterday, 1968) in ihren bekannten Büchern Zweigs Beziehung zum Judentum und zu Jerusalem nur flüchtig erwähnen. Vgl. Mark H. Gelber, Stefan Zweig, Judentum und Zionismus, Innsbruck, StudienVerlag (= Schriften des Centrums für Jüdische Studien, Bd. 24), 2014, S. 34ff.

10. Margarita Pazi, „Stefan Zweig: Europäer und Jude“, in Modern Austrian Literature, 14/3-4 (1981), S. 296. 
schon in seiner Jugend in der "Welt der Sicherheit" wahrgenommen haben musste. Der Literaturwissenschaftler Eduard Castle Mollineux sah seinen Zeitgenossen Zweig als „einen über den Rassen stehenden Europäer ${ }^{\text {"111 }}$, der gleichzeitig den sich verschlimmernden Judenhass und Herzls propagierten Zionismus ablehnt ${ }^{12}$. Auch Jacques Le Rider interpretiert Zweigs „unüberlegten Kosmopolitismus“ vor dem Ersten Weltkrieg und seinen „schwelgerischen Internationalismus“ in den zwanziger Jahren als eine „unbewusste Kompensation “"13.

Das Werk und das Leben Stefan Zweigs beweisen wiederholt, dass das multinationale Habsburgerreich, die Erziehung zur Weltoffenheit durch seine polyglotten Eltern und das Judentum für seine Entwicklung eine gleichwertige Rolle spielten. Im Vorwort zu seinem Erinnerungsbuch beschreibt Zweig sich „als Österreicher, als Juden, als Schriftsteller, als Humanist und als Pazifist ${ }^{\text {" }}{ }^{14}$, der sich Europa als eigentliche Heimat "erwählt" ${ }^{15}$ hatte. Dass Zweigs Europäertum und Judentum unlösbar verflochten sind, zeigt seine kulturelle Mittlerrolle - die Zohn und Pazi mit „der historischen Mission der Juden ${ }^{\text {“16 }}$ gekoppelt sehen - als Förderer des „europäischen Gedankens“ und als Übersetzer der Werke Verhaerens und Romain Rollands. Der Schriftsteller glaubte - wie man in seinem Briefwechsel mit Martin Buber liest -, dass einzelne Juden neben dem Judentum eine historische, weltbürgerliche Berufung hatten, und zwar die heilige Aufgabe, ihren übernationalen Geist und ihre universellen kulturellen Werte als ewig Vaterlandslose in den vom Nationalismus vergifteten europäischen Ländern zu verbreiten. Zweig lehnte jede Form von Verwurzelung und Auflösung ab, daher wünschte er sich nicht das Verschwinden der Juden durch die Assimilation oder durch den Zionismus - beides Alternativen eines Nationaljudentums

11. Eduard Castle, Deutsch-österreichische Literaturgeschichte. Ein Handbuch zur Geschichte der deutschen Dichtung in Österreich-Ungarn, Bd. 4, Wien, C. Fromme, 1937, S. 1730.

12. Zur frühen Beschäftigung des Autors mit dem Judentum (1900-1917) vgl. Stephan Resch, Stefan Zweig und der Europa-Gedanke, Würzburg, Königshausen \& Neumann, 2017, S. $81 \mathrm{ff}$.

13. Jacques Le Rider, „Stefan Zweig - Darstellung des Judentums in den Erzählungen der dreißiger und vierziger Jahre", S. 208.

14. Stefan Zweig, Die Welt von Gestern, S. 7.

15. Ebd., S. 8. In seinem ersten Selbstporträt Flüchtiger Spiegelblick identifiziert der Schriftsteller „vier Saiten seines Wesens" in sich selbst: „der Deutsche, der Jude, der Europäer, der Weltbürger“. C.V.-Zeitung, 20. Mai 1927, S. 1.

16. Harry Zohn, „Stefan Zweigs kulturelles Mittlertum: Ein jüdischer Charakterzug?“, in Bulletin des Leo Baeck Instituts 63 (1982), S. 19-31, hier S. 22. Vgl. auch Margarita Pazi, Stefan Zweig, S. 296. 
-, sondern er erhoffte sich ihre ewige Diaspora unter den Völkern der Erde. Sein Bonmot dazu lautete: „Durch Zerstreuung zur Erlösung ${ }^{\text {“17 }}$. In den Vaterlandslosen - einem Kapitel seiner Biographie des französischen Humanisten Romain Rolland (1921) - vermittelte der Autor seine Idee des europäischen Traums, wo ,jede Rasse [...] durch das Prononzierte ihres Wesens zur Bereicherung irdischer Vielfalt und damit zur Steigerung des Lebens notwendig ${ }^{18 “}$ war. Nach Zweig war das tiefste Wesen der Juden „Analyse und Zersetzung“. Ihre Vaterlandslosigkeit war die "Stechfliege“, „die den ,ruppigen Hornviehnationalismus" aus seinen geistigen Grenzen treibt: ihre Zersetzung ist Sprengmittel des schon Abgestorbenen, des ,ewig Gestrigen' und befördert neuen Geist, den sie selbst nicht zu schaffen vermögen“. Die Vaterlandslosen waren das Vorbild des zukünftigen „guten Europäers“"19.

Zweig war kein gläubiger Jude ${ }^{20}$. Wie der Autor in den ersten Seiten seiner Autobiographie erzählt, stammte er aus einer "guten“ jüdischen Familie der Doppelmonarchie, die sich durch „eine Flucht ins Geistige“ „von allen Defekten und Engheiten“ des östlichen orthodoxen Judentums befreit hatte, indem sie sich im Westen „an eine universale Kultur “'21 angepasst hatte. In Jerusalem war er nie, obwohl er in seinen Wanderjahren als begeisterter Globetrotter weltweit reiste ${ }^{22} .1908$ hatte er aber Palästina umschifft. Dies 'Umschiffen' ist im literarischen Schaffen des Autors als generelle Distanzhaltung zum "Jerusalem-Motiv“ anzunehmen, dem das untrennbar damit verknüpfte Motiv der ewigen Diaspora entgegengesetzt steht. In der Tat hat sich Stefan Zweig sein Leben lang mit dem Judentum, mit jüdischen Themen und jüdischer Geschichte und Tradition beschäftigt. Wegen seiner angeborenen „jüdi-

17. Zit. nach George Prochnik, Das unmögliche Exil, S. 143.

18. Stefan Zweig, „Romain Rolland“, in Ders., Gesammelte Werke, Düsseldorf, Null Papier Verlag, 2013, S. 4571.

19. Ebd.

20. 1881 wurde Stefan Zweigs Geburt mit dem hebräischen Namen Samuel im Geburtsbuch für die israelitische Cultusgemeinde in Wien eingetragen. Vermutlich wurde der Schriftsteller mit seinem jüdischen Vornamen am Sabbat und an Feiertagen aufgerufen. Vgl. Eva Plank, Ich will euch eine Zukunft und eine Hoffnung geben (Jer 29,11): Die biblische Prophetengestalt und ihre Rezeption in der dramatischen Dichtung Jeremias von Stefan Zweig, Göttingen, V\&R Unipress, 2018, S. 21.

21. Stefan Zweig, Die Welt von Gestern, S. 26-27.

22. Vgl. Matjaž Birk, „Reisen ist Rast in der Unruhe der Welt“. Fremdhermeneutische Einblicke in die Reisetagebücher von Stefan Zweig, Würzburg, Königshausen \& Neumann, 2016, S. 27. 
schen Sensibilität “'23 interessierte er sich von Jugend an für jüdische Thematiken und Autoren. Er kam in Kontakt mit jüdischen Schriftstellern und Intellektuellen, mit denen er sich über spezifisch jüdische Ideen wie biblische Stoffe, den Zionismus, das Ostjudentum, die jüdischen Sprachen und die jüdische Literatur, die bedrohte Existenz der europäischen Juden nach dem Ersten Weltkrieg und dann im Nationalsozialismus austauschte.

Mit der Zeit fühlte Zweig seine jüdische Identität als etwas Selbstverständliches ${ }^{24}$, wie seinen „Herzschlag ${ }^{“ 25}$. Hier soll der Versuch unternommen werden zu durchleuchten, wie Zweigs Diasporakonzept sich anhand seiner eher begrenzten jüdischen literarischen Produktion als "Raum der alternativen Ordnung" des menschlichen Zusammenlebens in der zeitgenössischen Realität in wechselnder Form einer Heterotopie - eines geistigen Jerusalems und eines übernationalen Europas - entwikkelte $^{26}$. Zweigs ästhetische Konstruktion der Diaspora erfolgte m. E. in einem fiktionalen Zwischenraum, wo die jüdische Literatur und Kultur gerade nicht durch nationalkulturelle Zugehörigkeit - egal ob deutsch oder jüdisch - bestimmt werden konnten, sondern wesentlich universal, kosmopolitisch, extraterritorial und transnational waren ${ }^{27}$. Seine jüdischen Novellen so wie sein Drama Jeremias (1917) lassen sich in der Tat weder in den Kanon der assimilativ-liberalen deutsch-jüdischen Literatur noch in jenen der dissimilativen zionistischen Literatur der jüdischen Renaissance einreihen. In der Epoche des postemanzipatorischen Antisemitismus konnte der kosmopolitische Schriftsteller dem Traum der deutsch-jüdischen Symbiose keinen Glauben mehr schenken, und er schloss sich auch nicht dem Zionismus an. 1936, als jede berufliche

23. Mark H. Gelber, „Judentum und jüdische Identität“, in Arturo Larcati, Klemens Renoldner, Martina Worgötter (Hg.), Stefan Zweig Handbuch, Berlin, de Gruyter, 2018, S. 755 .

24. Vgl. Gershon Shaked, „Die Gnade der Vernunft und die des Unglücks: Zweig und Roth - Ein Briefwechsel“, in Mark H. Gelber (Hg.), Stefan Zweig heute, New York Peter Lang (= New Yorker Studien zur Neueren Deutschen Literaturgeschichte 7), 1987, S. 141-159, hier S. 153.

25. Brief an Martin Buber vom 8. Mai 1916. Stefan Zweig, Briefe 1914-1919, hrsg. Knut Beck, Jeffrey B. Berlin, Natasha Weschenbach, Frankfurt/M., S. Fischer, 1998, S. 108.

26. Zum Thema Heterotopie vgl. Philip V. Bohlmann, „Jüdische Lebenswelten zwischen Utopie und Heterotopie, jüdische Musik zwischen Schtetl und Ghetto", in Lied und Populäre Kultur, 47. Jahrg. (2002), S. 37.

27. Zum Diasporakonzept vgl. Andreas B. Kilcher, „Diasporakonzepte“, in Hans Hotto Horch (Hg.), Handbuch der deutsch-jüdischen Literatur, Berlin/Boston, De Gruyter, 2016, S. 135-136. 
Beziehung mit Nazideutschland abgebrochen war, befürwortete Zweig dennoch eine eigenstaatliche Lösung für die von den Nationalsozialisten verfolgten Juden ${ }^{28}$. Keine Existenzform außerhalb des transnationalen Schriftraums der Diaspora wurde dagegen für sich selbst - sogar während des selbstgewählten Exils - in Betracht gezogen.

In seinen Memoiren bekennt Zweig sein Zugehörigkeitsgefühl zum Ostjudentum, da er sein „immanentes Ideal“ des „Aufstiegs ins

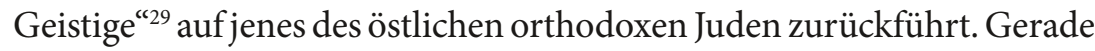
wegen seines „urjüdischen“ Willens zum Aufstieg ,in eine höhere kul-

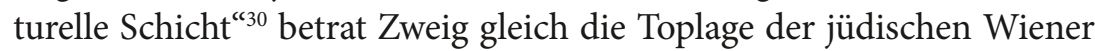
Szene. Der junge Literat trat in Verbindung mit der exklusiven JungWien-Gruppe, zu der akkulturierte jüdische Schriftsteller vom Rang Hofmannsthals, Schnitzlers, Beer-Hofmanns zählten. 1901 lernte er Theodor Herzl, den charismatischen Führer der zionistischen Bewegung und damals Feuilletonredakteur der Neuen Freien Presse kennen und wurde in den Kreis der Beiträger des Blattes aufgenommen, ohne sich jedoch dessen Zionismus anzueignen ${ }^{31}$. Die Begegnung mit Herzl und die Bekanntschaft in Berlin mit dem galizischen, zionistischen Künstler Mose Ephraim Lilien erweckten in Zweig ein gewisses Interesse für die Debatte der jungjüdischen Bewegung über Religion, Kunst und Politik, die unter den Juden der europäischen Diaspora ein jüdisches Bewusstsein und Selbstbewusstsein fördern wollte ${ }^{32}$. Wie es scheint, nahm der Schriftsteller oft an den jungjüdischen Diskussionsabenden teil, die unter der Ägide von Martin Buber an der Universität Wien stattfanden ${ }^{33}$. Gel-

28. Der Gedanke einer territorialen Lösung der Judenfrage des Schriftstellers steht in der Einleitung zum Werk Joseph Leftwichs What Will Happen to the Jews (1936). Mark H. Gelber, Judentum und jüdische Identität, S. 757. In einem undatierten, aber vermutlich im Mai 1938 verfassten Brief an Arnold Zweig erklärte sich der Autor gegen den Zionismus: „Auch Palästina war eine Sentimentalität, ein Antilogismus [...]. Palästina kann nicht mehr von dem verarmten und erschöpften Judentum weiter getragen und subventioniert werden $[\ldots]$ “. Wegen der dringenden Lage der bedrohten jüdischen Massen und der jüdischen Flüchtlinge hätte Stefan Zweig vielleicht eine pragmatische Entscheidung treffen wollen. Zit. nach Mark H. Gelber, Stefan Zweig, Judentum und Zionismus, S. 31.

29. Stefan Zweig, Die Welt von Gestern, S. 26.

30. Ebd.

31. Vgl. das Nachwort von Heinz Lunzer in Madaleine Rietra, Rainer Joachim Siegel (Hg.), "Jede Freundschaft mit mir ist verderblich“. Joseph Roth und Stefan Zweig. Briefwechsel 1927-1938, Göttingen, Wallstein, 2011, S. 579. Zu Zweigs Beziehung zum Zionismus siehe Mark H. Gelber, Stefan Zweig, Judentum und Zionismus, S. 57ff.

32. Vgl. Margit Dirscherl, „Stefan Zweig: Judentum und Zionismus bei Mark H. Gelber“, in The Modern Language Review, Vol. 110, 4 (2015), S. 1165.

33. Diese von Buber organisierten jungjüdischen Kulturabende waren ein wichtiger Teil des 
ber spricht von einer "time of flirtation with the movement “"34 während seiner letzten Universitätsjahre. Im Milieu der jüdischen Renaissance wurde der Schriftsteller nicht nur mit ihm bis dahin wenig bekannten religiösen Themen der Kunst und der Literatur vertraut, sondern auch mit ihrem künstlichen Versuch einer Mittlerfunktion zwischen der zionistisch geprägten jüdischen Kultur und der nicht-jüdischen Kultur, die Zweig das ästhetische Vorbild eines später selbst entwickelten europäischen Kulturtransfers lieferte. Wie Gelber bemerkt: „The jungjüdische Bewegung sought to package Jewish content, widely but Zionistically defined, in stylish and aesthetically conscious, non-Jewish artistic forms. In this sense, the movement aimed to bridge East and West ${ }^{\text {"35 }}$. Trotz seiner überzeugten Ablehnung der Hauptströmung von Herzls Politik entwickelte Zweig ein inniges Verhältnis zum Kulturzionismus, den er als „schöpferische Idee ${ }^{\text {“36 }}$ zur Wiederbelebung der jüdischen Kultur in der Diaspora lobte. Dass sich in seinem Bekannten- und Freundeskreis viele einflussreiche Kulturzionisten finden lassen, wie etwa Martin Buber und Berthold Feiwel ${ }^{37}$, erklärt eine gewisse unverkennbare zionistische Färbung in den ersten jüdischen Arbeiten Zweigs, die den Kulturzionisten sofort - doch vergeblich - Anlass zum Versuch gab, ihn als jungjüdischen Autor zu kanonisieren. Kein Wunder, dass im August 1901 seine Judennovelle Im Schnee in der von Buber und Feiwel herausgegebenen zionistischen Zeitschrift Die Welt ${ }^{38}$ (Wien) erschien, trotz Zweigs Aus-

kulturzionistischen Programms in Wien und Berlin am Anfang des 20. Jahrhunderts. Mark H. Gelber, Stefan Zweig, Judentum und Zionismus, S. 90-91.

34. Mark H. Gelber, „The jungjüdische Bewegung“, in Yearbook Leo Baeck Institute, 31, (1986), S. 105-119, hier S. 114.

35. Ebd., S. 118.

36. Der Gedanke einer territorialen Lösung der Judenfrage des Schriftstellers steht in der Einleitung zum Werk Joseph Leftwichs What Will Happen to the Jews (1936). Mark H. Gelber, Judentum und jüdische Identität, S. 757. In einem undatierten, aber vermutlich im Mai 1938 verfassten Brief an Arnold Zweig erklärte sich der Autor gegen den Zionismus: „Auch Palästina war eine Sentimentalität, ein Antilogismus [...]. Palästina kann nicht mehr von dem verarmten und erschöpften Judentum weiter getragen und subventioniert werden $[\ldots]$ “. Wegen der dringenden Lage der bedrohten jüdischen Massen und der jüdischen Flüchtlinge hätte Stefan Zweig vielleicht eine pragmatische Entscheidung treffen wollen. Zit. nach Mark H. Gelber, Stefan Zweig, Judentum und Zionismus, S. 31.

37. Mark H. Gelber, Stefan Zweig, Judentum und Zionismus, S. 97.

38. Die kleine Novelle Im Schnee wurde zusammen mit drei frühen Gedichten Zweigs, Trugland, Spinoza und Das Gericht, in Die Welt veröffentlicht. 1902 erschienen dieselben Werke im zionistischen Periodikum Jüdischer Almanach, in einer teilweise veränderten Ausgabe. Vgl. Mark H. Gelber, „The Impact of Martin Buber on Stefan Zweig“, in Modern Austrian Literature 14/3-4 (1981), S. 313-335, hier S. 315. Zum Verhältnis Stefan Zweigs zum Zionismus vgl. Jeffrey B. Berlin, „Response and Impression. Encountering Concepts 
sage in einem Brief an Franzos, dass der Novelle eine nationalistische Sendung fehle. Die Erzählung schildert das Schicksal einer Judengemeinde zur Zeit der mittelalterlichen Geißlerumzüge, die gegen ihren Willen eine deutsche Stadt in der Nähe zur polnischen Grenze verlassen muss. Während die Ostjuden Chanuka feiern, werden sie vor dem bevorstehenden Überfall der Flagellanten gewarnt. Die Dorfältesten entscheiden sich zur Flucht statt zum Widerstand. Und so flieht die ganze Gemeinschaft von „arme[n], zitternde[n], hilflose[n] Juden ${ }^{\text {“39 }}$ aus ihrer tödlich bedrohten Heimat. Ihr als Gotteswille angenommener Exodus führt sie aber nicht in ein Gelobtes Land, sondern in einen Schneesturm, wo sie den Tod finden. Der Autor stellt das Leid der wandernden Juden während der Vertreibung aus ihrer Heimat dar ${ }^{40}$, bietet aber keine zionistische Lösung für ihre ahasverische Existenz an. Trotz der romantisch mittelalterlichen Form hat er dennoch - wie Gelber pointiert feststellt - einen aktuellen historischen Aspekt dramatisch zum Ausdruck gebracht, der auf der Tagesordnung des Zionismus stand ${ }^{41}$. Zweigs Judenwanderschaft im Schnee deutet zweifellos die Massenmigrationen an, die osteuropäische Juden im späten 19. und frühen 20. Jahrhundert in Folge von Pogromen ${ }^{42}$ zur Vertreibung in die Vereinigten Staaten und in andere Länder oder zur Bildung jüdisch-nationaler Selbstverteidigung und zionistischer Bewegungen geführt hatten. Der tragische historische Hintergrund der Novelle sollte - nach einer eher gerechtfertigten kulturzionistischen Interpretation - die jüngere Generation der Juden Europas anspornen, die aussichtslose Opfermentalität der älteren durch eine einheitliche Vergeistigung des Lebens zu kontrastieren. Die Erzählung hat meiner Ansicht nach ein offenes Ende. Der halluzinative Traum des jungen sterbenden Protagonisten Josua, der zum letzten Mal den goldenen Leuchter brennen sieht, lässt an die ewige Wanderschaft als einzig rettende Hoffnung und geistige Kraft für das zukünftige Leben

of Judaism and Zionism in the Unpublished Correspondence between Martin Buber and Stefan Zweig (1902-1931)“, in Germanisch-Romanische Monatsschrift 30, 3 (2000), S. 333-360, hier S. 339.

39. Vgl. Alexander Schüler, „Die deutsch-jüdische Literatur der Wiener Moderne“, in Hans Otto Horch, Handbuch der deutsch-jüdischen Literatur, S. 316.

40. Ebd.

41. Mark H. Gelber, Stefan Zweig, Judentum und Zionismus, S. 95.

42. Zweig nimmt u. a. Bezug auf die Pogrome in der Ukraine, in Polen, in Litauen und Weißrussland nach der Ermordung des Zaren Alexander II. (1881), in Moskau (18911892), in Frankreich nach der Dreyfus-Affäre (1895-1898), und dann in Kishinev und Russland (1903). 
in der Diaspora denken, wie Zweig in späteren Werken (Jeremias, Der begrabene Leuchter, Die Welt von Gestern,) zeigt. Laut Margarita Pazi hat der Autor den tragischen Tod der jüdischen Flüchtlinge im Schneegestöber eher als eine Naturkatastrophe denn als eine dramatische Folge der "kalten" Gleichgültigkeit der europäischen Umwelt dargestellt ${ }^{43}$. Mir scheint dagegen, dass der Verfasser seine Geschichte im Licht der Menora und in der Wärme des hereinbrechenden Frühlings gipfeln lässt, um eine geistig kämpferische Alternative der Judenfrage darzulegen. Interessant erscheint diesbezüglich an Zweigs Einleitung zu Liliens Werk (E. M. Lilien. Sein Werk, 1903) die Kritik an dem sozialen Zionismus zugunsten der „schöpferischen“ Wendung zu einem jüdischen Vitalismus, der an den Ästhetizismus Bubers erinnert ${ }^{44}$ :

[Zionismus] hat das Judentum wieder bewußt gemacht; die schlafenden künstlerischen Werte geweckt, er hat in tausend Augen, die trostlos ins Dunkel starrten, das Sternbild einer realen Möglichkeit entzündet. Mag man die soziale Seite des Zionismus wie immer auch betrachten, mit dem merkwürdigen Lächeln gegenüber der Utopie oder dem schöpferischen Eifer der begeisterten - die tiefe ästhetische Schönheit dieses Krongedankens wird niemand leugnen können. Denn seit dem Sozialismus hat keine Idee soviel Ferne und Verlorene in ein Ziel zusammengebannt; mit Riesenkraft hat er aus einer wachsenden Zersplitterung und Entfremdung - da die Einheit der rituellen Religiosität langsam in die moderne Gottesidee zerbröckelte - eine kraftvolle Einheit gestaltet und das Nationale aus den scheckigen Hüllen der Nationalitäten befreit ${ }^{45}$.

Die „tausend Augen, die trostlos ins Dunkel starrten“ weisen auf die verhängnisvolle Hoffnungslosigkeit und Resignation der Juden in der Novelle Im Schnee auf, denen die befreiende Kraft der zionistischen Kunst und die (Buber'sche) „erziehende-kulturelle Funktion“46 der jüdischen Kultur gegenüberstand.

In der Erzählung findet man das wiederkehrende Motiv der Sehnsucht nach einem "guten Ort" (damit ist nach einem jiddischen Ausdruck der jüdische Friedhof bezeichnet), der - wie die Diaspora - das Moment der Zwischenräumlichkeit aufruft, die ihrerseits an das Merkmal der Heterotopie als einer Örtlichkeit „außerhalb aller Orte“ erinnert ${ }^{47}$. Das

43. Vgl. Margarita Pazi, Stefan Zweig, S. 297.

44. Vgl. Giuliano Baioni, Kafka. Literatur und Judentum, Stuttgart-Weimar, Metzler, 1994, S. 14.

45. Stefan Zweig, „Einleitung“, E. M. Lilien. Sein Werk, Berlin, Schuster \& Loeffler, 1903, S. 19.

46. Mark H. Gelber, The Impact of Martin Buber on Stefan Zweig, S. 317.

47. Vgl. dazu die Aussage von Michel Foucault über die Heterotopie des Friedhofs: „[...] Jede 
ist m. E. der potentielle Wendepunkt der Zweig'schen Novelle, der in anderen Arbeiten zum bedeutenden Ausgangspunkt der Umdeutung der modernen Diaspora zu einem ästhetisch-kosmopolitischen Modell jüdischer Moderne wird, das sein Vorbild in Heinrich Heine und Leopold Zunz hatte ${ }^{48}$. In der Wanderung der Juden nach Polen stellt Zweig in seiner Novelle zwischen Traum und Realität dar, dass - mit Zygmunt Baumann gesprochen - „in der Diaspora zu leben [...] gleichbedeutend mit einem Leben inmitten von Diasporas" ${ }^{\text {49 }}$ [ist]. Infolgedessen ist „jede diasporische Gemeinschaft [...] immer von einer tiefen Spaltung durchgezogen: räumlich zwischen Hier und Dort, emotional zwischen einem unmittelbaren Erleben und einer externen Bindung, gesellschaftlich zwischen kulturellen Eigenheiten und dem Hinauswachsen über diese Eigenheiten“. In den Worten von Isolde Charim [ist] „Diasporaexistenz [...] Existenz an und in Grenzen“, „die Erfahrung einer Spaltung “50. Auf dieses Thema komme ich noch einmal auf den folgenden Seiten zurück. An dieser Stelle sei gesagt, dass die Juden am Scheideweg zwischen einer Bejahung und einer Verneinung der Galuth stehen, dass sie sich für ein Leben in der geistigen extraterritorialen nationalen Existenz und ein Leben in dem Nationalismus

Heterotopie [hat] ein ganz bestimmtes Funktionieren innerhalb der Gesellschaft, und dieselbe Heterotopie kann je nach der Synchronie der Kultur, in der sie sich befindet, so oder so funktionieren. Als Beispiel nehme ich die sonderbare Heterotopie des Friedhofs $[\ldots]$ “. Nach Foucault lässt sich am Friedhof zeigen, inwiefern dies eine Heterotopie ist, die an „einen einzigen Ort mehrere Räume, mehrere Platzierungen“ zusammenzulegen vermag, „die an sich unvereinbar sind“. Der Friedhof kann als räumliche Inszenierung des Todes als eine Erscheinung nicht-genuin räumlicher Bedeutung aufgefasst werden. S. 40. Vgl. Michel Foucault, „Andere Räume“, in Karlheinz Barck u.a. (Hg.), Aisthesis. Wahrnehmung heute einer Ästhetik oder Perspektiven einer anderen Ästhetik, Leipzig, Reclam, 1992, S. 34-46, hier S. 40-41.

48. Mark H. Gelber stellt in diesem Zusammenhang fest, dass das Vorbild Zweigs für seine Novelle Im Schnee, die Werke der Anhänger des Vereins für Kultur und Wissenschaft des Judentums waren, wie etwa Heinrich Heine (Der Rabbi von Bacherach, 1840), Karl Emil Franzos (Die Juden von Barnow, 1877), Leopold von Sacher-Masoch (Polnische Geschichten, 1887, oder Neue Judengeschichten, 1881) mit ihrer Geschichten über die Flagellanten. Mark H. Gelber, The Impact of Martin Buber on Stefan Zweig, S. 321. Gerade die Schriftsteller der Kultur und Wissenschaft des Judentums plädierten für die Selbstbehauptung der jüdischen Identität in der Vielsprachigkeit und Plurikulturalität der Diaspora in der Moderne.

49. Zygmunt Bauman, „Leben in der Diaspora“, in Isolde Charim, Gertraud Auer Borea (Hg.), Lebensmodell der Diaspora, Bielefeld, transcript, 2012, S. 95-103, hier S. 95.

50. Zit. nach Ulrich Engel, „Grenzen als ,Loci theologici ${ }^{-}$Skizzen zu einer nachkonziliaren Diasporatheologie“, in Petrus Bsteh, Brigitte Proksch, Diaspora - monotheistische Weise der Weltpräsenz. Die Bedeutung von Charismen und religiösen Gemeinschaften für die Ortskirche, Wien, LIT Verlag, 2017, S. 67. 
der Assimilation oder des Zionismus entscheiden müssen. Der in der Novelle „ungelöste“ Ahasverismus - der in dem Traum Josuas optimistisch als ewige Diaspora erscheint - ist meiner Ansicht nach eine Kritik des Autors an den obengenannten Nationaltendenzen.

Rückblickend möchte ich darauf verweisen, dass die zweite Veröffentlichung von Zweigs Novelle Im Schnee in dem 1902 erstmals herausgegebenen Jüdischen Almanach sogar als eine kontrastierende Antwort auf die konkrete zionistische Utopie Herzls gesehen werden kann, die im Roman Altneuland (1902) in dem verwirklichten Traum der Rückkehr des jüdischen Volks nach Jerusalem dargestellt wird. Gleichzeitig widersetzte sich der Autor mit seiner „scheinbar“ akritischen Darstellung der von ihrem schmerzlichen Schicksal geeinigten Brüderschaft der ostjüdischen Gemeinde den Kulturzionisten, die gegen das Leiden der Juden in der Diaspora mit ostentativem „Zorn“ in der Publizistik protestierten ${ }^{51}$.

Die Faszination Stefan Zweigs für den Kulturzionismus so wie seine schwankende geistige Freundschaft mit Buber war nur zeitweise intensiv, und zwar während der früheren Definition seiner jüdischen Identität in der Wiener Kulturwelt ${ }^{52}$. Gelber behauptet, dass Zweigs Interesse an jüdischen Themen zur Jahrhundertwende zu keiner anhaltenden künstlerischen oder intellektuellen Beschäftigung mit der jüdischen Vergangenheit führte, weil seine jüdischen Kenntnisse begrenzt und oberflächlich waren und weil der Autor allmählich Interesse für den europäischen Humanismus eines Rollands und eines Verhaerens aufgebracht hatte ${ }^{53}$.

Stefan Zweig war m.E. nie gleichgültig gegenüber der Geschichte seines Volks. Nennenswert ist diesbezüglich seine persönliche Stellung-

51. In Martin Bubers Zeitschrift Der Jude fand eine radikalzionistische Diasporakritik statt. Eine drastische Galuthverneigung vertraten Abraham Schwadron und Jakob Klatzkin, um nur zwei Beispiele zu nennen. Als unglückliches Ergebnis der Emanzipation und Assimilation sahen sie Selbstverlust, Selbstaufgabe, Selbstverleugnung. Auf nicht jüdischer Seite waren die assimilierten Juden durch den postemanzipatorischen Antisemitismus in Verdacht gekommen, weil sie gesehen wurden als „diejenigen, die ohne Hindernisse am weitesten in alle Canäle der Gesellschaft und des politischen Gemeindelebens eindringen“. Das Problem der Moderne überhaupt sah Eugen Düring (Die Judenfrage als Racen-Sitten- und Culturfrage, 1881) in der „Einstreuung des Racenjudentums in die Fugen und Spalten unserer nationalen Behausungen“. Die gewünschte Lösung der Judenfrage war die Auflösung ihres Nationalen. Vgl. Andreas B. Kilcher, Diasporakonzepte, S. 139-140.

52. Vgl. Jeffrey B. Berlin, Response and Impression, S. 340.

53. Vgl. Mark H. Gelber, Stefan Zweig, Judentum und Zionismus, S. 98. 
nahme zu seinen Arbeiten und zu jüdischer Literatur, die der Schriftsteller 1937 Alfred Wolf mit diesen Worten erklärte: „Und da wir eben Juden sind, und es nicht verleugnen, so wird in sich schon dies Werk einen jüdischen Charakter annehmen ${ }^{\text {"55 }}$. Wenn man auch nicht von einer Allgegenwärtigkeit der Bibel in der literarischen Produktion von Zweig sprechen kann, war dennoch die Bibel für den Autor ein literarisches Werk und ein bevorzugtes System zentraler Autorität. Zweig war tatsächlich nicht an den religiösen Aspekten der Bibel interessiert, sondern an den künstlerischen. Als Schriftsteller spürte er in der Heiligen Schrift das Geheimnis der Schöpfung Gottes, das sich in jeder Kunst versteckt, wie der Verfasser 1939 in seinem Essay Das Geheimnis des künstlerischen Schaffens schreibt: „Gefühl eines Überirdischen, eines Göttlichen, wenn irgendwo zuerst ein Nichts war und plötzlich ein Etwas ist, das vorher nicht gewesen ist " ${ }^{\text {"55 }}$. In diesem Essay bezieht sich der Autor nur einmal explizit auf das Alte Testament, und zwar als er den künstlerischen Schaffensprozess mit dem „Kampf Jakobs mit dem Engel“ vergleicht: „jene[m] ewigen Kampf des Künstlers, der zu dem Engel der Vollendung sagt: Ich lasse dich nicht, du segnest mich denn!" ${ }^{\times 6}$ (1. Mose 32, 23-33). Der Kampf des Patriarchen mit dem Engel am Jabbok findet statt, als er den Namen Israel erhält. Die Schaffung wird als das zähe Ringen eines "geschaffenen Wesens", das durch die Bewältigung der künstlerischen Aufgabe, durch die Überwindung seiner selbst und der entgegenstehenden Widerstände auf eine höhere Stufe gehoben und implizit zu Israel wird ${ }^{57}$.

An dieser Stelle möchte ich kurz zwei Arbeiten Zweigs erwähnen, in denen religiöse Stoffe verhandelt werden. Die erste ist Die Wanderung (1901), die Herzl 1902 in der Neuen Freien Presse herausbrachte. Die Wanderung, die der Autor beschreibt, ist diesmal keine Massenflucht, sondern er zeigt die Stationen eines eifrigen, sehnsüchtigen Pilgers aus Judäa - vielleicht eines Juden, einer verschleierten Projektion des Schriftstellers -, dem es nicht gelingt, Jesus Christus, beziehungsweise den Erlöser und Messias, zu sehen, weil er sich unterwegs seiner Wol-

54. Zit. nach Alfred Wolf, Jüdische Elemente im Werke Stefan Zweigs, University of Cincinnati, 1937. Unveröffentlichte Arbeit von Alfred Wolf im Zweig-Archiv, Fredonia, S. 11.

55. Stefan Zweig, „Das Geheimnis des künstlerischen Schaffens“, in Ders., Buchmendel. Erzählungen, S. 348.

56. Ebd., S. 369.

57. Zum Biblischen in Stefan Zweigs Werk vgl. Armin Eidherr, „Biblische Stoffe und Motive", in Arturo Larcati, Klemens Renoldner, Martina Worgötter (Hg.): Stefan-ZweigHandbuch, S. 635-641, hier S. 635. 
lust hingibt. Als er endlich in Jerusalem ankommt, erkennt er unter den gekreuzigten Verbrechern den Erlöser nicht. Vermutlich wollte Zweig mit dieser kurzen Erzählung das uralte Motiv der Wanderung durch die „Unrast“, die „wachsende Unruhe“, den „eilenden Wanderschritt“"58 schildern, die die Existenz und den Charakter des jüdischen Volks bestimmt hat. Bemerkenswert ist die Tatsache, dass die Novelle nach ihrem Erscheinen 1904 in der Novellenreihe Die Liebe der Erika Ewald (1904) nicht mehr gedruckt wurde. In einer anderen Novelle aus der gleichen Sammlung, Die Wunder des Lebens (1903), greift Zweig auf die religiöse Geschichte der Juden im Europa des 16. Jahrhundert zurück. Die Reformationskämpfe in Flandern bilden den historischen Hintergrund der Handlung an. Ein christlicher Maler ist beauftragt worden, ein Altarbild im Dom Antwerpens fertigzustellen, das eine Madonna mit einem nackten Säugling darstellen soll. Als Gottesmutter wird Esther, ein jüdisches Mädchen gewählt, das Christen hasst, weil seine Eltern in einem Pogrom ermordet worden sind. Trotzdem beginnt Esther während des Malprozesses, Mutterliebe zu dem Kind zu entwikkeln. Als das Bild fertig ist, geht sie wiederholt in die Kirche, um die gemalte Mutter-Kind-Szene zu bewundern, die eine symbolische Übertragung einer vorbildlichen Koexistenz zwischen Christen und Juden darstellt. Eines Tages wird das Gotteshaus von einer tobenden Menge geplündert und zerstört. Das Judenmädchen wird erstochen, während sie versucht, das Altarbild vor den Plünderern zu schützen. Zweig, der 1902 gestanden hatte, dass er „immer in einem fremden Verhältnis zur katholischen Kirche gestanden ${ }^{\text {"59 }}$ habe, der aber auch kein gläubiger Jude war, lässt hier die Figuren in ihrer eigenen Religion und Kultur ausschließlich in der Kunst friedlich miteinander umgehen. Die Erzählung ist mit Zeichnungen seines zionistischen Freunds Lilien illustriert worden, ein Zeichen, dass Zweig mit den Mitgliedern des jungjüdischen Kreises über die durch jüdische Assimilationsbestrebungen nicht lösbare Situation der europäischen Juden desillusioniert debattierte ${ }^{60}$.

Um die Jahrhundertwende, als Zweig seine Identität zu bestimmten versuchte, fand er eine geistige Verbundenheit in der kulturzionistischen

58. Stefan Zweig, "Die Wanderung", in Ders., Die Liebe der Erika Ewald, Berlin, Fleischel \& Co, 1904, S. 7-9.

59. Stefan Zweig, Die Wunder des Lebens, in Ders., Buchmendel, S. 319.

60. Vgl. Christine Rechberger, „Die Wunder des Lebens“, in Arturo Larcati, Klemens Renoldner, Martina Worgötter (Hg.), Stefan Zweig Handbuch, S. 184-188, hier S. 185. Vgl. auch Volker Henze, Jüdischer Kulturpessimismus und das Bild des alten Österreich im Werk Stefan Zweigs und Joseph Roths, Heidelberg, Winter, 1988, S. 202-203. 
schöpferischen Idee, die „ein Renaissancegedanke, eine Heimatidee “61 war. Die innere Distanz des Schriftstellers nicht nur gegenüber dem Zionismus Herzls, sondern auch gegenüber jenem Bubers wird mit der Zeit zu einer geistigen und auch zu einer literarischen Stellungnahme gegen jede Form von Nationalismus, wie diese Aussage in einem Brief vom 6. November 1922 an den Verleger Hans Rosenkranz zeigt: „Wie groß war die Idee [des Zionismus], wie rein, solange sie noch ganz Traum war, ungemengt mit Politik und Soziologie. [...] Dazu hatte ich nicht die Kraft, ich hing zu sehr an der Kunst, an der Welt, an der Welt als ganzem, nur mich bloß einer Nation hinzugeben ${ }^{\text {"62 }}$.

Beim Ausbruch des Ersten Weltkriegs trat bei Stefan Zweig eine existentielle Krise auf, die ihn zu einer neuen Auseinandersetzung mit der jüdischen Identität führte. Während der ersten Kriegsmonate war er ,ja

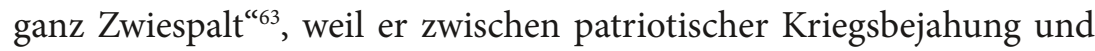
kosmopolitischem Pazifismus hin- und hergerissen war. Der Schriftsteller musste sich dem Judentum und dem biblischen Stoff annähern, um die Widersprüche der eigenen hybriden Identität aufzulösen. Während seiner militärischen Dienstpflicht im Wiener Kriegsarchiv wurde der Schriftsteller als Titularfeldwebel im Juni 1915 mit einem Sonderauftrag betraut: Er erhielt den Befehl, nach Galizien zu reisen, um sich über die Verhältnisse in diesem österreichischen Kronland aus erster Hand zu informieren ${ }^{64}$. Besonders die jüdische Diaspora in den galizischen Kriegsgebieten, wo das Schicksal der vertriebenen Juden viel dramatischer als „seit den Tagen Nebukadnezars und der Zerstörung des Tempels ${ }^{\prime 65}$ aussah, erinnerte den Schriftsteller an die ostjüdische Gemeinde der eigenen Novelle Im Schnee. Es ist sicherlich kein Zufall, dass Zweig in den schwierigsten Kriegstagen in Galizien sein jüdisches Drama entwarf, wie man in der Welt von Gestern lesen kann: „Mitten im

61. Stefan Zweig, Ephraim Mose Lilien. Das Geheimnis des künstlerischen Schaffens: Essays, hrsg. Knut Beck,, Frankfurt/M., Fischer, 1984, S. 51.

62. Zit. Nach Mark H. Gelber, „Stefan Zweigs verspätete Bekehrung zum Judentum“, in Bulletin des Leo Baeck Instituts 63 (1982), S. 7.

63. Tagebuch, am 28. Dezember 1914. Stefan Zweig, Tagebücher, hrsg. Knut Beck, Frankfurt/M., S. Fischer, 1984, S. 127. Zum Thema Stefan Zweig und der Erste Weltkrieg vgl. Paola Paumgardhen, „Stefan Zweig: la guerra dentro“, in Marino Freschi, Paola Paumgardhen, Grande Guerra e Mitteleuropa. L'Austria-Ungheria, Acireale-Roma, Bonanno editore, 2015, S. 75-108, hier S. 85.

64. Hartmut Müller, Stefan Zweig, Hamburg, Rowohlt, 1988, S. 57.

65. Stefan Zweig, „Warum nur Belgien, warum nicht auch Polen? Eine Frage an die Neutralen", in Ders., Die schlaflose Welt, Frankfurt/M., S. Fischer, 1983, S. 52-67, hier S. 59. 
Kriege, indes die andern sich noch, voreilig triumphierend, gegenseitig den unfehlbaren Sieg bewiesen, warf ich mich schon in den untersten Abgrund der Katastrophe und suchte den Aufstieg. Aber unbewußt hatte ich, indem ich ein Thema der Bibel wählte, an etwas gerührt, das in mir bisher ungenützt gelegen: an die im Blut oder in der Tradition dunkel begründete Gemeinschaft mit dem jüdischen Schicksal" ${ }^{\text {“66 }}$. Zweig beschäftigte sich mit dem biblischen Stoff intensiv vom Frühjahr 1915 bis zu Ostern 1917, und sein Drama erschien noch während seines Militärdienstes im Insel Verlag.

Der Autor wollte das Sonderschicksal der Juden mit einer pazifistischen Aussage verknüpfen, um seinem zerstreuten Volk eine neue Hoffnung in der Kriegsdiaspora zu schenken. Die Gestalt des Propheten Jeremias, des biblischen Mahners, schien ihm dafür geeignet, die „seelische Superiorität des Besiegten ${ }^{\text {“67 }} \mathrm{zu}$ behaupten. Als der Krieg verlorengeht, erweist sich der ,vergebliche Warner "68 als moralischer Helfer seines erniedrigten Volks. Er tröstet den König Zedechia und die Kriegsüberlebenden, die Jerusalem verlassen und in die Fremde ziehen müssen. Die Niederlage weckt die seelische Kraft der Besiegten. Das historische Jerusalem ist verloren, aber das geistige Jerusalem zieht mit ihnen und begleitet sie in die Diaspora. Auf ihrer ewigen Wanderschaft werden die Juden nie verloren sein, denn, wie Jeremias vorankündigt: „Man kann das Unsichtbare nicht besiegen! Man kann Menschen töten, aber nicht den Gott, der in ihnen lebt. Man kann ein Volk bezwingen, doch nie seinen Geist ${ }^{\text {“69 }}$.

Dass Zweigs Selbstsuche in vollem Gang war, wird von seiner erneuten Ablehnung jedes nationalen Gedankens bestätigt, jetzt wieder in den Tagen des kriegerischen Patriotismus wie früher in seiner Begegnung mit den Zionisten. Beim Schreiben des Dramas hatte der Verfasser vermutlich einen neuen Aspekt des Judentums entdeckt, den er für sich selbst als identitätsstiftend ansah. Der selbstbewusste Auszug des verbannten Volks aus Jerusalem in die Diaspora zeigt, dass es eine geistige statt einer geographischen Heimat hat. Wie Eva Plank richtig feststellt, wird im Jeremias nicht nur das Leiden des Gottesvolkes dargelegt, sondern auch die Ausrichtung Zweigs als Jude der Diaspora. In dem neunten Bild der Tragödie, Der ewige Weg, will Zweig nicht die Rück-

66. Stefan Zweig, Die Welt von Gestern, S. 288-289.

67. Ebd, S. 288.

68. Ebd.

69. Stefan Zweig, Jeremias, in Ders., Tersites. Jeremias. Zwei Dramen, Gesammelte Werke, hrsg. Knut Beck, Frankfurt/M., S. Fischer, 1982, S. 327. 
kehr nach Jerusalem als erstrebtes Ziel des Exodus zeigen, vielmehr ist der Auszug aus Jerusalem ein Aufbruch in die Welt ${ }^{70}$. Jerusalem wird zu einem geistigen Ort, der den realen, materiellen Tempel durch die ewige, unsichtbare Gegenwart Gottes ersetzt. Am 24. Januar 1917 schrieb der Autor einen Brief an Martin Buber, wo sein „jüdisches" Europäertum und Kosmopolitismus überdeutlich wird:

$\mathrm{Da}$ ich die Diaspora liebe und bejahe als den Sinn seines Idealismus [des Judentums], als seine weltbürgerliche allmenschliche Berufung. Und ich wollte keine andere Vereinigung als im Geist, in unserem einzigen realen Element, nie in einer Sprache, in einem Volke, in Sitten, Gebräuchen, diesen ebenso schönen als gefährlichen Synthesen. Ich finde den gegenwärtigen Zustand den großartigsten der Menschheit: dieses Eins-Sein ohne Sprache, ohne Bindung, ohne Heimat, nur durch das Fluidum des Wesens ${ }^{71}$.

Zweig konnte die Krisensituation des Kriegs überwinden, indem er die jüdische Heimatlosigkeit als einen befreienden Übernationalismus deutete und das jüdische Leiden als moralische Erlösung ansah. Damit hatte er sich ein für alle Mal „der Realpolitik ${ }^{\text {“72 }}$ des Zionismus widersetzt. Der Autor meinte, dass es besser für die Juden war, sich von der Politik fern zu halten, und nun wollte er seine unpolitische Haltung offen erklären, wie es in diesem Brief an seinen zionistischen Freund Abraham Schwadron zu lesen ist:

Für mich ist es der Ruhm und die Größe des jüdischen Volkes, das einzige zu sein, das nur eine geistige Heimat, ein ewiges Jerusalem anstrebt, während er zu Wiederkehr ins reale Palästina graviert. Für mich ist es die Größe des Judentums, übernational zu sein, Ferment und Bindung aller Nationen in seiner eigenen Idee, er wünscht die jüdische Nation, und ich sehe in jedem Nationalismus die Gefahr der Entzweiung, des Stolzes, der Eingrenzung und der Eitelkeit ${ }^{73}$.

Der „ewige“ Weg stellt am Schluss des Dramas das im Exil beginnende Schicksal des jüdischen Volkes und seine ununterbrochene Wanderschaft in der Welt dar. Da Zweig das wandernde Leben der Juden zwischen unbegrenzt offener Zukunft und fernster Vergangenheit schildert, lässt sich daraus folgern, dass der Autor keine reale Rückkehr nach Palästina im Blick hatte.

70. Vgl. Eva Plank, Ich will euch eine Zukunft und eine Hoffnung geben, S. 80.

71. Stefan Zweig, Briefe an Freunde, hrsg. Richard Friedenthal, Frankfurt/M., S. Fischer, 1984, S. $68 \mathrm{f}$.

72. Brief an Martin Buber vom 25. Mai 1917, in Stefan Zweig, Briefe 1914-1917, S. 142.

73. Stefan Zweig, Briefe an Freunde, S. 71. 
Wenn die Diaspora der eigentliche Nährboden für den Zionismus war, deutete Zweig sie dagegen zur historischen, „höchsten Mission ${ }^{\text {“74 }}$ um. Seinen biblischen Propheten ließ er dem unglücklichen, vaterlandslosen Volk Israel den Sinn ihres Leidens mit diesen Worten erklären: „Ich sehe den Gott darin. Seine Prüfung nur ist diese Stunde, so lasset sie uns bestehen! "755. Im Zeichen eines radikalen Monotheismus stand die Aussage Jeremias', dass „Gott als die letzte Ursache für alles Geschehen in seiner Schöpfung, also auch des Leids ${ }^{\text {" }}{ }^{76} \mathrm{zu}$ sehen war. Die harte Prüfung der Juden wird als unbegrenzte Liebe zu dem unsichtbaren Gott der Auserwählten begründet:

Damit wir ihn erkennen, sendet Gott uns die Prüfung. Andern Völkern ist klein' Zeichen und gering' Erkennen gegeben, in Hölzern und Steinen meinen sie des Ewigen Gesicht zu erschauen. Doch unser Gott, unserer Väter Gott, ein verborgener Gott ist er, und erst in der Tiefe des Leidens werden wir seiner gewahr, nur in der Prüfung tut er sich auf seinen Erwählten. Segen, wem sie begegnet, denn was wäre Israel unter den Völkern, prüfte es nicht ewig sein Gott? Wen er liebet, den stößt er hinab in die Tiefe des Lebens, daß er ihn erprobe, und, ihr Brüder, immer hat Gott sein Volk geliebt, immer hat er es hinabgestoßen ${ }^{77}$.

Noch 1936 beschrieb er in seiner im Londoner Exil verfassten Judennovelle Der begrabene Leuchter das immerwährende Wandern und die ständigen Verfolgungen der Juden als die geistigen Wurzeln der verbrüdernden Kraft des kollektiven Leidens in dem tragischen Schicksal der Heimatlosigkeit. Dem weisen Rabbi Elisier legt der Autor eine Rede in den Mund, in der er auch auf das unveränderbare Schicksal der Diasporaexistenz des Judentums als zu beachtenden Gotteswillen darin eingeht:

Ein alter Weg ist es, den wir gehen, mein Kind, schon unsere Väter und Vorväter sind ihn gegangen. Denn ein Wandervolk sind wir gewesen unendlich viele Jahre lang und sind es wieder geworden, und vielleicht sogar, wer weiß es, ist es unser Geschick, daß wir es bleiben für ewige Zeit. Nicht wie die andern Völker haben wir Erde unter unserem Schlafen zu eigen, nicht wächst uns im eigenen Felde Samen und Frucht. Nur mit wandernden Füßen gehen wir über die Länder, und in fremde Scholle sind unsere Gräber getan ${ }^{78}$.

74. Stefan Zweig, Briefe 1914-1917, S. 355.

75. Stefan Zweig, Jeremias, S. 310.

76. Ebd.

77. Ebd.

78. Stefan Zweig, „Der begrabene Leuchter“, in Ders., Legenden, hrsg. Knut Beck, Stockholm, Beermann-Fischer, 1949, S. 107. 
Zweig legt ein kollektiv leidendes Gottesvolk dar, das im Gottesglauben in der Zerstreuung vereint bleibt: „Aber zerstreut, wie wir sein mögen, und zwischen die Furchen geworfen wie Unkraut von Morgen bis Mitternacht dieser Erde, sind wir doch Volk geblieben, ein einziges und einsames unter den Völkern, durch unseren Gott und den Glauben an ihnn ${ }^{\text {“"79. }}$. Die jüdische Neigung zur geistigen statt zur physischen Kraft, die Zweig seinem Volk zuschrieb, beweist, dass der Verfasser dabei der Tolstoi'schen Tradition folgte, der zufolge der Geist wertvoller als die körperliche Macht war. Das erklärt Zweigs/Jeremias' Vorliebe für den „Schwache[n]“, für den „Ängstliche[n]“, der „in der Stunde der Niederlage sich meist als der einzige erweist, der sie nicht nur erträgt, sondern sie bemeistert ${ }^{\text {“80 }}$. Bis zum Ende seines Lebens blieb Zweig, obwohl schwankend in seiner Einstellung, seiner geistigen und ästhetischen Existenz in der Diaspora verhaftet. Stephan Resch vertritt die Meinung, dass Zweigs Diasporakonzept eine moralische und idealistische Perspektive anbot, doch es unterschätzte die Gefahr für sein heimatloses Volk und für ihn selbst, weiterhin auf das Wohlwollen der einzelnen Nationalstaaten angewiesen zu sein. Zweigs Jeremias entstand im Milieu des spätliberalen Bürgertums, als der Schriftsteller aus einer gesicherten sozialen Stellung die Vorzüge der Staatenlosigkeit noch preisen konnte ${ }^{81}$. Die oftmals verfolgten und gesellschaftlich ausgegrenzten Juden konnte Zweig durch sein Diasporakonzept selbstverständlich nicht trösten, wie ihm Martin Buber am 17. Mai 1917 in Bezug auf seinen Jeremias schrieb: „Es geht mir ganz und gar nicht ein, daß diese Worte in diesem Augenblick für diese Menge der Trost seien ${ }^{\text {“82. }}$. Durch sein „persönlichstes“ und „privatestes ${ }^{\text {“83 }}$ Werk neben dem Erasmus (1934), versuchte der pazifistische Autor nicht nur dem Wandervolk zu helfen, sondern auch ihm selbst, da er es in „erbittertstem Widerstand gegen die Zeit ${ }^{\text {“84 }}$ geschrieben hatte.

Joseph Kastein meint, dass Jeremias nicht die Selbstbehauptung des Judentums Zweigs darstellt, sondern eher eine Aneignung des biblischen Motivs seitens des Dramatikers ist. Ich stimme diesbezüglich mit der Meinung Garrins überein, dass der Autor trotz einer gewissen Distanz zu dem Schicksal der Ostjuden sowohl in Jeremias als auch in

79. Ebd.

80. Stefan Zweig, Die Welt von Gestern, S. 288.

81. Vgl. Stephan Resch, Stefan Zweig und der Europa Gedanke, S. 89.

82. Unveröffentlichter Brief. Zit. nach Jeffrey B. Berlin, Response and Impression, S. 355.

83. Stefan Zweig, Die Welt von Gestern, S. 288.

84. Ebd., S. 291. 
Der begrabene Leuchter das Leid der Juden mitfühlte ${ }^{85}$. Er identifizierte sich mit seinem Propheten, der das Geschick der Juden lapidar darstellt: „Nicht zum Frieden sind wir erwählet unter den Völkern: Weltwanderschaft ist unser Zelt ${ }^{\text {“86 }}$. Eugen Relgis, Zweigs rumänischer Übersetzer, hält den Schriftsteller für einen „great European“, der sich nie von seinem Volk, von dessen historischem Schicksal, dessen Ethik, und dessen Idealen von Frieden, Gerechtigkeit und Freiheit trennte ${ }^{87}$. Wie schon 1927 Richard Specht bemerkte, ist es nur teilweise gerechtfertigt, wenn Zweigs bedeutendstes jüdisches Werk Jeremias als rein pazifistische Kundgebung des Autors interpretiert wird, da man in dieser Dichtung „einen dramatischen Emporschrei gegen die verabscheute Lüge und die verbrecherisch grausame Brutalität des Krieges und der Völkermentalität, [...] zum erstenmal ein[en] Appell an die Seele Europas ${ }^{{ }^{188}}$ hören kann. Ein Jahr später schrieb auch Erwin Rieger, dass Jeremias sich ,in Deutschland als erster tragischer Protest gegen das Unvermeidliche erhob $^{\text {“89. }}$. Dass Zweig trotz allem unfähig war, sich in politische oder staatsmännische Zusammenhänge zu vertiefen, zeigen diese bedeutenden Zeilen aus einem Brief an Max Brod vom 30. Juni 1926, wo er seine Einstellung zu begründen versucht:

Manchmal glaube ich daran und frage in mich hinein, ob man nicht tätiger sein solle. Aber man darf nichts halb tun: ein europäischer Aktivismus erfordert den ganzen Menschen. [...] Wir, Sie und ich, haben Augenblicke des Ausbruchs, des Willens ins Reale. Im wesentlichen aber wohnen wir im Raum (oder im Kerker) der geistigen Welt... [...]. Darum glaube ich, wir können nur (wie ich's versuchte) anregen, aufrufen, erinnern. Organisieren, agitieren müssen die andern. Ich habe oft geschwankt zwischen beiden Welten - aber die andere ist für uns die wirkliche, nicht diese der Compromisse und Zweideutigkeiten $[\ldots]^{90}$.

Man kann sicher nicht verschweigen, dass der Schriftsteller sich hier irrte, da Brod im Gegensatz zu Zweig schon seit 1917 zionistisch aktiv war. Wir können trotzdem diese Worte Zweigs als seine „unpolitische“ Idee einer geistigen und ästhetischen Diaspora interpretieren.

85. Vgl. Stephan H. Garrin, "Stefan Zweig's Judaism”, in Modern Austrian Literatur, 14/3-4 (1981), Special Stefan Zweig Issue, S. 271-289, hier S. 285.

86. Stefan Zweig, Jeremias, S. 201.

87. Vgl. Stephan H. Garrin, Stefan Zweig's Judaism, S. 285.

88. Richard Specht, Stefan Zweig. Versuch eines Bildnisses, Leipzig, Spamerschen, 1927, S. 13.

89. Erwin Rieger, Stefan Zweig. Der Mann und das Werk, Berlin, J. M. Spaeth, 1928, S. 76.

90. Zit. nach Stephan H. Garrin, Stefan Zweig's Judaism, S. 301. 
Als die Juden ihr geliebtes Land in "gewaltige[r] Bewegung ${ }^{\text {“91 }}$ verlassen, beginnt Jeremias mit bebender Stimme seinen poetischen Aufruf zum Aufbruch, indem er den Titel „Leidenvolk“ durch „Wandervolk“ ersetzt: „Auf, ihr Besiegten, / Rüstet zur Reise! / Wandervolk, Gottesvolk, welterwähltes, / Hebe dein Herz! ${ }^{\mid 92}$. Der Prophet ermuntert das wandernde Volk, einen letzten Blick auf die Heimatstadt zu werfen, die er - obwohl zerstört - als ,ewige Stadt ${ }^{“ 93}$ bezeichnet. Ewig bleibt Jerusalem im ständigen Sich-Erinnern an die verinnerlichte Stadt. „Wer glaubt“ - so Jeremias zu seinem leidenden Volk - „schaut immer Jerusalem ${ }^{\text {“94. }}$. Der Glaube an einem unsichtbaren Gott (das Gesetz) löst Jerusalem (den Tempel) als realen Ort der Verehrung Gottes ab. Als die Chaldäer, die Sieger, die Judäer verzückt ihre Heimat verlassen sehen, zeigen sie ein gewisses Verständnis für den jüdischen Glauben: „Ein Geheimnis

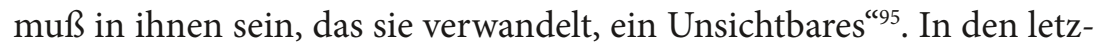
ten Versen des Dramas verwandelt der Autor dank dem Glauben an Gott den Fluch der ewigen Wanderschaft zum Segen des „Wandern[s] in alle Ewigkeiten ${ }^{\text {“96. }}$. Diese Gottesgewissheit, welche die Urväter und das Volk noch während des Mittelalters im Gegensatz zu den Juden des zwanzigsten Jahrhunderts stärkte, fühlte der Autor der Welt von Gestern noch als die einzige - doch unbefriedigende - Antwort auf den ständigen Zweifel der Juden: „Warum wir alle?“. Noch in den letzten Tagen seines Exils und Lebens überlegte der Schriftsteller: „Aber vielleicht ist es gerade des Judentums letzter Sinn, durch seine rätselhaft überdauernde Existenz Hiobs ewige Frage an Gott immer wieder wiederholen, damit sie nicht völlig vergessen werde auf Erden ${ }^{\text {(997. }}$.

Die Stelle, in der Zweig die unaufhörliche Diaspora der Juden durch (den Dichter) Jeremias segnet, erinnert uns unmittelbar an den 1934 für Die Wahrheit verfassten Artikel von Joseph Roth Der Segen des ewigen Juden, wo der ostjüdische Schriftsteller seine religiöse und politische Idee der Diaspora gegen die Nationalsozialisten und die Zionisten äußerte: „Das Wandern ist kein Fluch, sondern ein Segen ${ }^{\text {“98. Daraus }}$

91. Stefan Zweig, Jeremias, S. 319.

92. Ebd.

93. Ebd.

94. Ebd., S. 321.

95. Ebd., S. 326.

96. Ebd.

97. Stefan Zweig, Die Welt von Gestern, S. 484.

98. Joseph Roth, Werke. Das journalistische Werk, 3 Bde., Klaus Westermann (Hg.), Köln, Kiepenheuer \& Witsch, 1989-1981, Bd. 3, S. 532. 
könnte man schließen, dass auch das Drama Zweigs eine kraftvolle politische Aussage trägt. Obwohl Zweig bei seinem Grundsatz blieb, keine politischen Artikel zu verfassen, finden wir doch seine politischen Gedanken in seinen literarischen wie in seinen privaten Schriften. Im Februar 1918 behauptete er in einem Brief an Buber: „Denn ich bin ganz klar und entschlossen, je mehr sich im Realen den Traum zu verwirklichen droht, der gefährliche Traum eines Judenstaates mit Kanonen, Flaggen, Orden, gerade die schmerzliche Idee der Diaspora zu lieben, das jüdische Schicksal mehr als das jüdische Wohlergehen "999.

Die ästhetische Diaspora Stefan Zweigs lässt sich in die DiasporaLiteratur des zwanzigsten Jahrhunderts eingliedern, die nicht nur ein Kunstprogramm hatte, sondern auch ein politisches. Man denke an das Diaspora-Programm von Alfred Wolfenstein (1922) gegen die Nationalisierung der Juden durch Assimilation wie Zionismus und an seine spirituelle Sendung in der Moderne als europäischer und kosmopolitischer Dichter. Man denke an Anton Kuhs (1921) Kritik an den Leitmustern jüdischer Identität in der Moderne: Assimilation und Zionismus, beides Ausdrücke jüdischen Opportunismus. Dagegen forderte Kuh ein oppositionelles Judentum, das sich in der genuinen Lebensform der Extraterritorialität erkennen sollte. Andere jüdische Expressionisten, wie Yvan Goll oder Ludwig Rubiner sprachen in anarchistischem Ton von Heimatlosigkeit und Gemeinschaft. Als Gegner des Zionismus entwickelte der humanistische Schriftsteller Lion Feuchtwanger in der Weimarer Republik einen politisch-ästhetischen Begriff der Diaspora gegen den nochmals sich verschärfenden Nationalismus Anfang der dreißiger Jahre. Während die Zionisten aus Europa nach Zion ausziehen wollten, forderte Feuchtwanger eine umgekehrte Richtung von Zion nach Europa, um das nomadische Existential zum Modell zu erheben ${ }^{100}$.

In einem Kapitel der Welt von Gestern, dessen Titel Sonnenuntergang lautet, schreibt Zweig: „Nie sind die Menschen so viel gereist wie in diesen Jahren. [...] Ich konnte mit stärkerem Nachdruck und breiterer Wirkung für die Idee werben, die seit Jahren die eigentliche meines Lebens geworden: für die geistige Einigung Europas"101. Das Diasporische erscheint auch als positives Momentum einer conditio judaica, das ein Vorbild für die Moderne sein kann, indem es sich dem Nationalismus widersetzt. Im Werk Zweigs - wie im Werk seines

99. Stefan Zweig, Briefe 1914-1919, S. 202.

100. Vgl. Andreas B. Kilcher, Diasporakonzepte, S. 142-145.

101. Gemeint ist das Jahrzehnt 1924-1933. Stefan Zweig, Die Welt von Gestern, S. 371. 
galizischen Freundes Joseph Roth - spiegelt die Diasporaexistenz der Juden die Übernationalität der im Ersten Weltkrieg untergegangenen Doppelmonarchie wider. In seinem journalistischen Werk Jedermann ohne Pass (1934) äußert Roth seine Idee, dass die nomadischen Schriftsteller keine Außenseiter sind, sondern die Vorposten der Zukunft, da die ganze Erde Vaterland ist ${ }^{102}$. Dies war auch das politische Diasporakonzept Zweigs.

In den Prosawerken zwischen den Weltkonflikten thematisiert Zweig auf einer makrotextuellen Ebene das dramatische Schicksal der Juden im Exil, wobei er einerseits an das ewige Wandern (Rahel rechtet mit Gott,1927) und andererseits an den freien Geist (Untergang eines Herzens, 1927 und Buchmendel, 1929) appelliert. In den letzten zwei Arbeiten stellt der Autor durch die unüberwindbare Spaltung zwischen assimiliertem Westjudentum und orthodoxem Ostjudentum den Untergang des entgeisterten Abendlandes dar. Buchmendel erzählt die Geschichte Jakob Mendels, eines bibliophilen galizischen Juden, der in dem kosmopolitischen Café Gluck Wiens zum übernationalen Weltbürger wird. Wegen der Nationalisierung Europas zur Zeit des Ersten Weltkriegs wird ihm der Zutritt in das Kaffeehaus verboten. Bekanntermaßen waren Cafés im fin-de-siécle Wien internationale Verkehrsplätze, heterotopische Zwischenorte der jüdisch-europäischen Kultur, transnationale Oasen, die Zweig später während seiner USA-Reise vermisste $^{103}$.

Die Vertreibung des Talmud-Gelehrten und Bücher-Sauriers Mendel aus dem Café Gluck kann als dramatische Vorwegnahme des geistigen Schicksals Europas und des Schriftstellers selbst gesehen werden. Nach einer Hausdurchsuchung nach Waffen durch die Polizei verließ Zweig 1934 Österreich freiwillig. Er empfand das als eine unheilbare Verletzung seiner Integrität. In seiner letzten Legende, Der begrabene Leuchter, stellt vielleicht - wie Eva Plank bemerkt - das Begraben des Leuchters, der stilistisch ein Symbol des Lebensbaumes ist, das Begraben von Zweigs humanistischen Idealen dar ${ }^{104}$. Die Menora versinnbildlicht die Vereinigung des Allgemeinmenschlichen und des Jüdischen, die der Autor in dem Europa der Diktaturen als unvereinbar sah.

Die selbstgewählte Diaspora außerhalb Europas führte Zweig zuerst in die USA, dann nach Brasilien. Im brasilianischen Exil verfasste er

102. Vgl. Andreas B. Kilcher, Diasporakonzepte, S. 147.

103. Zur Heterotopie der Cafés vgl. Michel Foucault, Andere Räume, S. 38-39.

104. Vgl. Eva Plank, Ich will euch eine Zukunft und eine Hoffnung geben, S. 69. 
seine „europäischen Memoiren“ Die Welt von Gestern. Das Buch schildert durch eine melancholische Reise in die Vergangenheit und in die Fremde Europas die letzte Wanderung des kosmopolitischen Wiener Juden Stefan (Samuel) Zweig, dem seine eigentliche Wahlheimat verloren war. Der rastlose Wanderer behauptete noch einmal die "Schwäche des Besiegten“: „Aber ich beklagte das nicht; gerade der Heimatlose wird in einem neuen Sinne frei, und nur der mit nichts mehr Verbundene braucht auf nichts mehr Rücksicht zu nehmen "105. Gleichzeitig wollte Zweig noch einmal sein humanistisches Ideal einer übernationalen Identität erklären, die in der Erzählung Buchmendel in Frage gestellt war. In der posthum erschienen Montaigne-Biographie (1942) rechtfertigte der Autor sein eigenes Schicksal. Das Leben Montaignes kann tatsächlich als Widerspiegelung jenes von Zweig gesehen werden: Er war Sohn einer jüdischen Mutter und dennoch nicht einer Rasse und eines Vaterlandes, sondern Weltbürger ${ }^{106}$.

Die Weltliteratur blieb für Stefan Zweig im Exil der „andere Ort“. 1933 möchte der europamüde Stefan Zweig den ihm teuersten Besitz, seine Briefsammlung, der Bibliothek in Jerusalem übermachen. Am 11. Dezember 1933 schrieb er dem damaligen Direktor der Jewish National Library in Jerusalem, Professor Hugo Bergmann:

Darf ich Sie bitten, diesen Brief streng vertraulich zu behandeln. Ich möchte, daß nicht ein Wort davon an die Öffentlichkeit käme. Ich weiß nicht, ob ich meinen Hausstand aufrecht erhalte bei den kommenden Verhältnissen in Österreich und mehr als das Materielle machen mir gewisse persönliche Dinge Sorgen. Z. B. meine Privatkorrespondenz. [...] Ich habe da eine Auslese gemacht [...] und sie umfaßt [...] so ziemlich alles Wesentliche unserer Epoche, Hauptmann, Rolland, Verhaeren, Einstein, Dehmel, Freud, Maeterlinck, Herzl, Valery, Rathenau, Richard Strauss, Joyce, Gorki, Thomas Mann, etc. [...] Es stellt ein recht rundes Bild der Weltliteratur der letzten dreißig Jahre dar ${ }^{107}$.

Stefan Zweig stellte sich eine literarische Diaspora in dem heterotopischen Zwischenraum einer jüdischen Bibliothek in Jerusalem vor, wo die übernationale europäische Kultur wiederaufleben könnte. Auf rastloser Wanderschaft hatte er Palästina immer nur umwandert. Jerusalem galt in der Kunstauffassung des kosmopolitischen Schriftstellers als literarischer Raum, in dem Übergänge geschaffen wurden, die zur Überwindung von Grenzziehungen auffordern.

105. Stefan Zweig, Die Welt von Gestern, S. 7-8.

106. Vgl. Eva Plank, Ich will euch eine Zukunft und eine Hoffnung geben, S. 75.

107. Zit. nach Margarita Pazi, Stefan Zweig: Europäer und Jude, S. 306-307. 